quatrième série-tome $41 \quad$ fascicule 4 juillet-aồt 2008

$$
\begin{aligned}
& \text { ANNALES } \\
& \text { SCIENTIFIQUES } \\
& \text { de } \\
& \text { L'ECOLE } \\
& \text { NORMALE } \\
& \text { SUPÉRIEURE }
\end{aligned}
$$

Frédéric TOUZET

Feuilletages bolomorphes de codimension un

dont la classe canonique est triviale 


\title{
FEUILLETAGES HOLOMORPHES DE CODIMENSION UN DONT LA CLASSE CANONIQUE EST TRIVIALE
}

\author{
PAR FRÉDÉRIC TOUZET
}

RÉsumÉ. - We give a description of Kähler manifolds $M$ equipped with an integrable subbundle $\mathcal{F}$ of $T M$ of rank $n-1(n=\operatorname{dim} M)$ under the assumption that the line bundle Dét $\mathcal{F}$ is numerically trivial. This is a sort of foliated version of Bogomolov's theorem concerning Kähler manifolds with trivial canonical class.

Abstract. - Nous décrivons les variétés kählériennes compactes $M$ de dimension complexe $n$ dont le fibré tangent admet un sous-fibré holomorphe intégrable $\mathcal{F}$ de rang $n-1$ dont le fibré déterminant Dét $\mathcal{F}$ est à première classe de Chern nulle. Ce résultat peut en quelque sorte être considéré comme un avatar feuilleté du théorème de Bogomolov concernant les variétés kählériennes à fibré canonique numériquement trivial.

\section{Introduction}

Parmi les variétés kählériennes compactes $M$, celles dont le fibré canonique est numériquement trivial sont caractérisées par le remarquable résultat suivant, dû à $\mathrm{F}$. Bogomolov :

THÉORÈME $1.1([2,1])$. - Supposons que $c_{1}(M)=0$; alors $M$ admet un revêtement (étale) fini $\tilde{M}$ qui est le produit d'un tore par une variété de Calabi-Yau.

Rappelons qu'une variété kählérienne est dite de Calabi-Yau si elle est compacte, simplement connexe et admet une forme volume holomorphe, c'est-à-dire une forme holomorphe de degré maximal partout non nulle.

Suivant le fameux théorème de Yau ([Ya]), on peut munir une telle variété d'une métrique Ricci-plate dont l'holonomie induit une décomposition en produit de sous-variétés de Calabi-Yau irréductibles, c'est-à-dire dont le fibré tangent n'est pas holomorphiquement décomposable. 
Considérons une variété complexe $M$ munie d'un feuilletage holomorphe régulier de codimension un. Ceci correspond à la donnée d'un sous-fibré intégrable $\mathcal{F}$ de rang $n-1 \mathrm{du}$ fibré tangent $T M\left(n=\operatorname{dim}_{\mathbb{C}} M\right)$.

Il y a naturellement deux fibrés en droites holomorphes associés à $\mathcal{F}$, le fibré normal et le fibré canonique du feuilletage qui sont respectivement $N_{\mathcal{F}}=\frac{T M}{\mathcal{F}}$ et $K_{\mathcal{F}}=$ Dét $\mathcal{F}^{*}=$ $\wedge^{n-1} \mathcal{F}^{*}$ où $\mathcal{F}^{*}$ désigne le dual de $\mathcal{F}$.

Par adjonction, on hérite de l'isomorphisme

$$
N_{\mathcal{F}}^{*} \otimes K_{\mathcal{F}}=K_{M}
$$

où $K_{M}$ désigne comme à l'habitude le fibré canonique de la variété $M$.

Dans cet article, on s'intéresse à la situation suivante : $M$ est kählérienne compacte et est munie d'un feuilletage $\mathcal{F}$ dont la classe canonique est numériquement triviale, i.e.

$$
c_{1}\left(K_{\mathcal{F}}\right)=0 .
$$

Cette configuration est clairement réalisée par les trois exemples décrits maintenant :

i) à revêtement fini près, $M$ est le produit d'un tore par une variété de Calabi-Yau et $\mathcal{F}$ est obtenu en tirant en arrière un feuilletage linéaire de codimension 1 sur le premier facteur par la projection canonique;

ii) $M$ est une fibration rationnelle au-dessus d'une variété à première classe de Chern nulle (et par suite décrite par le théorème de Bogomolov) et le feuilletage $\mathcal{F}$ est transverse aux fibres;

iii) le feuilletage $\mathcal{F}$ est donné par une fibration en hypersurfaces à première classe de Chern nulle.

Nous affirmons que cette liste est exhaustive :

THÉORÈME 1.2. - Soit M une variété kählérienne compacte munie d'un feuilletage holomorphe régulier de codimension 1 à fibré canonique numériquement trivial; alors ce feuilletage est décrit par l'un des exemples (non mutuellement exclusifs) i),ii) ou iii) ci-dessus.

Ces résultats ne sont guère surprenants si l'on a en vue les conjectures d'abondance. En effet, ces dernières prévoient que le feuilletage $\mathcal{F}$ coïncide avec la fibration d'Itaka-Kodaira de la variété lorsque celle-ci n'est pas uniréglée, ce qui correspond effectivement au point iii) et laisse présager que cette description reste valide lorsque $\mathcal{F}$ est singulier.

Toujours dans l'esprit des conjectures d'abondance, on peut motiver notre travail par l'étude de variétés possédant certaines propriétés numériques.

Considérons par exemple une variété projective $M$ à fibré canonique nef de dimension de Kodaira numérique égale à 1 , c'est-à-dire telle que $c_{1}^{2}(M)=0$ et $c_{1}(M) \neq 0$. Géométriquement, cette dernière égalité suggère (dans un sens volontairement approximatif!) l'existence de directions d'annulation de la courbure du fibré canonique qui, dans quelques situations favorables pourraient être incarnées par un feuilletage à classe canonique (numériquement) triviale correspondant à l'exemple iii) ci-dessus.

Cet article est organisé comme suit. 
Dans la section 2 est d'abord examiné le cas (facile) où la variété $M$ est elle-même à classe canonique numériquement triviale. En fait, ce cas réapparaîtra de façon récurrente dans la discussion globale et va correspondre à l'exemple i) du théorème 1.2.

Nous construisons ensuite une classe $\alpha$ dans la cohomologie de Dolbeault canoniquement attachée au feuilletage $\mathcal{F}$. Cette opération peut être effectuée pour n'importe quel feuilletage holomorphe régulier de codimension 1 . Si l'on revient maintenant à nos hypothèses $\left.\left(c_{1}\left(K_{\mathcal{F}}\right)=0\right)\right)$, on montre que l'annulation de cette classe $\alpha$ nous renvoie au cas précité $\left(c_{1}(M)=0\right)$.

A contrario, sa non-annulation introduit une structure géométrique supplémentaire sur $M$, à savoir un feuilletage holomorphe en courbes $\mathcal{C}_{\omega}$, éventuellement singulier, mais en position très spéciale relativement à $\mathcal{F}$ :

$$
\mathcal{C}_{\omega} \text { est transverse à } \mathcal{F}
$$

ou

$$
\mathcal{C}_{\omega} \text { est tangent à } \mathcal{F} \text {. }
$$

Le reste de l'article est consacré à l'étude de ces deux configurations possibles.

Nous traitons dans la section 3 le cas $« \mathcal{C}_{\omega}$ transverse à $\mathcal{F} »$. C'est à ce niveau que vont apparaître la plupart des ingrédients de la preuve du théorème principal.

Précisons un peu comment les choses s'articulent.

Hormis la situation où $\mathcal{C}_{\omega}$ est un feuilletage en courbes rationnelles, ce qui correspond à l'exemple ii) du théorème 1.2, on peut construire un courant $T$ (lisse) invariant par holonomie de $\mathcal{F}$ «induit » par la métrique «naturelle » sur les feuilles de $\mathcal{C}_{\omega}$ suivant que celles-ci sont hyperboliques ou paraboliques. En particulier, le fibré canonique $K_{M}$ est pseudo-effectif et sa classe de Chern est représentée par $T$. Dans ces conditions, $-T$ peut s'incarner comme courbure de Ricci d'une certaine métrique kählérienne. Cette métrique qui est, d'une certaine façon, canoniquement associée au feuilletage $\mathcal{F}$ dote ce dernier de propriétés de parallélisme.

Ceci permet de préciser la décomposition de De Rham de $M$ (plus exactement celle de son revêtement universel), puis de constater que les feuilles de $\mathcal{F}$ sont nécessairement fermées (dans le cas $c_{1}(M) \neq 0$ ).

Dans la quatrième et dernière section, les deux feuilletages $\mathcal{F}$ et $\mathcal{C}_{\omega}$ sont supposés être tangents.

On montre dans un premier temps que la présence du feuilletage auxiliaire $\mathcal{C}_{\omega}$ entraîne la pseudo-effectivité du fibré canonique de la variété ambiante $M$.

De là, on déduit l'existence d'un courant positif invariant par holonomie de $\mathcal{F}$ et représentant la classe canonique de $M$. La situation est donc très similaire à celle étudiée dans la section 3 à ceci près que le courant est susceptible de présenter des problèmes de régularité. Cependant, le pseudo-groupe d'holonomie de $\mathcal{F}$ conserve suffisamment de «bonnes » propriétés (au sens où c'est un pseudo-groupe de Lie) et, modulo une analyse au cas par cas, cette observation suffit à conclure.

Ce travail s'appuie, d'une part, sur des résultats de géométrie des feuilletages (Brunella, Kellum) et, d'autre part, sur quelques théorèmes classiques de géométrie riemannienne, en particulier ceux de Yau (conjectures de Calabi), Cheeger et Gromoll (variétés à courbure de Ricci positive ou nulle). 


\section{Feuilletage en courbes associé}

\subsection{Remarque préliminaire quand $c_{1}(M)=0$}

À revêtement fini près, la variété $M$ est le produit d'un tore par une variété de Calabi-Yau (théorème 1.1). De plus, la formule d'adjonction 1.1 implique que $\mathcal{F}$ est défini par une forme holomorphe (éventuellement à valeurs dans un fibré plat) qui est donc identiquement nulle en restriction à toute sous-variété simplement connexe de $M$. Par suite, $\mathcal{F}$ est nécessairement un feuilletage «linéaire» sur $M$ au sens de l'exemple i) donné dans l'introduction.

\subsection{Classe de cohomologie $\alpha$ associée à $\mathcal{F}$}

Considérons un recouvrement ouvert $\left(U_{i}\right)_{i \in I}$ de $M$ suffisamment fin tel que sur chaque ouvert de la famille, les feuilles de $\mathcal{F}$ soient données par les niveaux d'une submersion holomorphe

$$
f_{i}: U_{i} \rightarrow \mathbb{C} \text {. }
$$

Sur chaque intersection $U_{i} \cap U_{j}$, les différentielles des $f_{i}$ sont liées par la relation

$$
d f_{i}=g_{i j} d f_{j}, g_{i j} \in \mathcal{O}^{*}\left(U_{i} \cap U_{j}\right)
$$

où le cocycle multiplicatif $g_{i j}$ représente le fibré normal du feuilletage $N_{\mathcal{F}}$.

Le faisceau $\mathcal{F}_{\infty}$ des germes de $(1,0)$ formes différentielles lisses tangentes $\mathcal{F}$ est fin, ce qui assure l'existence de sections $\omega_{i} \in \mathcal{F}_{\infty}\left(U_{i}\right)$ vérifiant sur $U_{i} \cap U_{j}$ :

$$
\omega_{i}-\omega_{j}=\frac{d g_{i j}}{g_{i j}} .
$$

Puisque les $\omega_{i}$ sont de la forme

$$
g_{i} d f_{i}
$$

où $g_{i} \in \mathcal{C}^{\infty}\left(U_{i}\right)$, on obtient par différentiation que

$$
\bar{\partial} g_{i}=g_{i j}{ }^{-1} \bar{\partial} g_{j}
$$

On constate ainsi que la collection des $\bar{\partial} g_{i}$ définit une classe $\alpha$ du groupe de cohomologie $H_{\bar{\partial}}^{0,1}\left(N_{\mathcal{F}}{ }^{*}\right)$.

En combinant isomorphisme de Dolbeault et dualité de Serre, on obtient que

$$
H_{\bar{\partial}}^{0,1}\left(N_{\mathcal{F}^{*}}{ }^{*}\right) \simeq H_{\bar{\partial}}^{0, n-1}\left(N_{\mathcal{F}} \otimes K_{M}\right)
$$

et par la formule d'adjonction (1.1)

$$
H_{\bar{\partial}}^{0, n-1}\left(N_{\mathcal{F}} \otimes K_{M}\right)=H_{\bar{\partial}}^{0, n-1}\left(K_{\mathcal{F}}\right) .
$$

Cette construction fonctionne quelles que soient les propriétés numériques du fibré $K_{\mathcal{F}}$.

En revanche, il faut invoquer sa platitude (ce qui est bien notre hypothèse principale) pour pouvoir en déduire, par symétrie de Hodge, l'isomorphisme

$$
H_{\bar{\partial}}^{0, n-1}\left(K_{\mathcal{F}}\right) \simeq H_{\bar{\partial}}^{n-1,0}(\text { Dét } \mathcal{F}) .
$$

$4^{\mathrm{e}}$ SÉRIE - TOME $41-2008-\mathrm{N}^{\mathrm{o}} 4$ 
2.2.1. Le cas $\alpha=0$. - En reprenant les notations précédentes, il existe sur chaque ouvert $U_{i}$ une fonction lisse $\varphi_{i}$ telle que

$$
\bar{\partial} g_{i}=\bar{\partial} \varphi_{i}, \varphi_{i}=g_{i j}{ }^{-1} \varphi_{j} \text { sur } U_{i} \cap U_{j}
$$

On en déduit alors que

$$
\alpha_{i} d f_{i}-\alpha_{j} d f_{j}=\frac{d g_{i j}}{g_{i j}}
$$

où $\alpha_{i}=g_{i}-\varphi_{i}$ est holomorphe sur $U_{i}$, en conséquence de quoi le fibré $N_{\mathcal{F}}{ }^{*}$ est muni d'une connexion holomorphe.

Puisque la variété ambiante est kählérienne, ceci implique la platitude de $N_{\mathcal{F}}{ }^{*}$ (donc de $K_{M}$ ) et permet de conclure par la remarque préliminaire 2.1 .

2.2.2. Le cas $\alpha \neq 0$. - Par la série d'isomorphismes (2.1), (2.2), (2.3) décrite en fin de section 2.1, la classe de Dolbeault $\alpha$ détermine une $n-1$ forme holomorphe $\omega$ non identiquement nulle sur $M$ à valeurs dans Dét $\mathcal{F}$. Localement, une telle forme a pour argument un multivecteur de degré $n-1$ et de ce point de vue, elle s'interprète naturellement comme une section holomorphe du fibré trivial $K_{\mathcal{F}} \otimes$ Dét $\mathcal{F}$. En particulier, la restriction de $\omega$ à $\mathcal{F}$ est soit identiquement nulle, soit partout non dégénérée. Notons $\mathcal{C}_{\omega}$ le feuilletage en courbe (éventuellement singulier) associé à $\omega$.

En résumé, il existe un feuilletage en courbes holomorphe $\mathcal{C}_{\omega}$ défini par le noyau d'une section holomorphe non triviale $\omega$ de $\Omega^{n-1} \otimes$ Dét $\mathcal{F}$ tel qu'on ait l'alternative suivante :

a) $\mathcal{C}_{\omega}$ et $\mathcal{F}$ sont partout en position transverse (en particulier, $\mathcal{C}_{\omega}$ est régulier),

b) $\mathcal{C}_{\omega}$ est tangent au feuilletage $\mathcal{F}$.

Ces deux cas vont être étudiés séparément dans les deux sections à venir.

\section{Le feuilletage $\mathcal{C}_{\omega}$ est supposé partout transverse à $\mathcal{F}$}

\subsection{Métrique « induite» $\operatorname{par} \mathcal{C}_{\omega}$}

Examinons d'abord le cas où $\mathcal{C}_{\omega}$ admet une feuille rationnelle. Du théorème de stabilité de Reeb, il résulte que toutes les feuilles sont rationnelles. Le feuilletage $\mathcal{F}$ est donc construit par une suspension de fibre $\mathbb{P}^{1}$ au-dessus d'une variété à première classe de Chern nécessairement nulle. Il s'agit donc de l'exemple ii) du théorème 1.2. Notons que l'holonomie de $\mathcal{F}$ est ici obtenue comme représentation de l'extension d'un groupe fini par un groupe abélien libre dans le groupe de Mœbius.

Si l'on exclut ce dernier cas de figure, il est établi dans [6] qu'un feuilletage en courbe $\mathcal{C}$ transverse à une distribution en hyperplan $D$ sur une variété kählérienne compacte est parabolique ou hyperbolique : toutes les feuilles sont uniformisées, soit exclusivement par la droite complexe, soit exclusivement par le disque unité. En outre, la distribution est en fait intégrable (c'est un feuilletage).

Dans le cas parabolique, on montre dans [6] que le fibré cotangent $T_{\mathcal{C}}{ }^{*}$ du feuilletage en courbes est plat. Dans notre contexte, cela signifie que $N_{\mathcal{F}}{ }^{*}\left(\simeq\right.$ à $T_{\mathcal{C}_{\omega}}{ }^{*}$ par tranversalité) est plat dès que $\mathcal{C}_{\omega}$ est parabolique et nous sommes à nouveau dans la configuration décrite dans la remarque 2.1 (exemple i), du théorème 1.2. 
Quand $\mathcal{C}=\mathcal{C}_{\omega}$ est hyperbolique, on peut construire (loc.cit.) sur le fibré normal $N_{\mathcal{F}}$ une métrique hermitienne invariante par holonomie de $\mathcal{F}$ et dont la restriction aux feuilles de $\mathcal{C}_{\omega}$ coïncide avec la métrique de Poincaré. Puisque $N_{\mathcal{F}}$ s'identifie à $T_{\mathcal{C}_{\omega}}$, on récupère par dualité $\operatorname{sur} N_{\mathcal{F}}{ }^{*}$ une métrique hermitienne sur $N_{\mathcal{F}}{ }^{*}$ dont la forme de courbure $\Omega$ est une $(1,1)$-forme fermée lisse, semi-positive et invariante par holonomie de $\mathcal{F}$; en particulier $\Omega$ s'annule dans la direction de $\mathcal{F}$.

\subsection{Preuve du théorème 1.2}

$\mathrm{Au}$ vu de la section 3.1, il reste à traiter le cas où $\mathcal{C}_{\omega}$ est hyperbolique, ce qui sera supposé ici.

Proposition 3.1. - La variété $M$ peut être munie d'une métrique kählérienne g qui induit sur chaque feuille de $\mathcal{F}$ une métrique Ricci plate.

De plus, on peut choisir cette métrique de telle sorte que $\tilde{M}$ (le revêtement universel de $M$ ) se scinde holomorphiquement et isométriquement (pour la métrique pull-back) sous la forme $\tilde{N} \times \mathbb{D}$. Cette décomposition de $\tilde{M}$ est compatible avec celle de $T M$ en ce sens que $T \tilde{N}$ et $T \mathbb{D}$ (vus comme sous-fibrés de TM) sont respectivement pull-back de $\mathcal{F}$ et $\mathcal{C}_{\omega}$.

Enfin, $\pi_{1}(M)$ agit diagonalement par isométries sur le produit $\tilde{M}=\tilde{N} \times \mathbb{D}$.

Démonstration. - Remarquons d'abord que la forme $-\Omega$ est un représentant de la classe anticanonique $c_{1}(M)$, eu égard à l'équivalence numérique de $N_{\mathcal{F}}{ }^{*}$ et $K_{M}$.

D'après le théorème de Yau ([14]), on en déduit l'existence sur $M$ d'une métrique kählérienne $g$ dont la courbure de Ricci vérifie l'égalité

$$
\operatorname{Ricc}(g)=-\Omega \text {. }
$$

En chaque point de $M$, le tenseur de Ricci est donc donné par une forme semi-négative. De plus, par hypothèse de platitude, il existe sur $M$ une collection de sections locales holomorphes $\xi_{U}$ de Dét $\mathcal{F}$ ne s'annulant pas et multiplicativement liées par un cocycle de module 1. En particulier, l'ensemble des $\Delta\left(\left\|\xi_{U}\right\|^{2}\right)$ et $\left\|\nabla\left(\xi_{U}\right)\right\|^{2}$ se recollent en une fonction globale définie sur $M$ ( $\nabla$ désigne ici la connexion induite par $g$ sur les tenseurs holomorphes de type $(n-1,0))$. Dans ces conditions, l'inégalité de Bochner ([10, p. 57]) s'énonce dans les termes suivants :

$$
\Delta\left(\left\|\xi_{U}\right\|^{2}\right) \geq\left\|\nabla\left(\xi_{U}\right)\right\|^{2} .
$$

Par suite, on obtient que $\nabla\left(\xi_{U}\right)=0$ pour tout ouvert $U$ du recouvrement. En d'autres termes, le fibré $\mathcal{F}$ est parallèle. Un résultat classique de géométrie kählérienne implique alors que son orthogonal est holomorphe ([9, th. 8.1, p. 172]). Quitte à réappliquer les résultats de [6], on peut conclure que ce dernier définit un feuilletage hyperbolique. La proposition 3.1 résulte alors du classique théorème de décomposition de De Rham joint au fait que $\operatorname{Ricc}(g)$ est identiquement nulle en restriction à $\mathcal{F}$.

Précisons maintenant la structure du premier facteur $\tilde{N}$ dans la décomposition du revêtement universel.

Proposition 3.2. - La variété $\tilde{N}$ se scinde (holomorphiquement et isométriquement) sous la forme $\mathbb{C}^{k} \times V$ où $V$ est une variété de Calabi-Yau.

En outre, $\pi_{1}(M)$ agit diagonalement par isométries sur le produit $\tilde{M}=\mathbb{C}^{k} \times V \times \mathbb{D}$. 
La preuve repose sur le résultat suivant de Cheeger et Gromoll.

ThÉORÈMe 3.3 ([7]). - Soit $M$ une variété lisse simplement connexe admettant une métrique riemannienne complète de courbure de Ricci positive ou nulle; alors $M$ se décompose isométriquement sous la forme $\mathbb{R}^{k} \times V$ où $V$ est une variété ne contenant pas de droite géodésique.

Rappelons qu'une droite géodésique est une géodésique

$$
\gamma:]-\infty,+\infty[\rightarrow M
$$

telle qu'à chaque instant $t, t^{\prime} \in \mathbb{R}$, le segment $\gamma_{\mid\left[t, t^{\prime}\right]}$ soit minimal.

Preuve de la proposition 3.2. - Puisque la décomposition de De Rham d'une variété kählérienne coïncide avec celle de la variété réelle sous-jascente ([9, th. 8.1, p. 172]), on obtient que $\tilde{N}$ se scinde holomorphiquement et isométriquement sous la forme $\mathbb{C}^{l} \times V$.

Par construction, le groupe fondamental de $M$ agit diagonalement par isométries sur le produit $\tilde{M}=\mathbb{C}^{l} \times V \times \mathbb{D}$. Il reste à prouver que $V$ est compacte; supposons par l'absurde que ce ne soit pas le cas. La contradiction recherchée résulte alors de l'argument suivant repris verbatim à Cheeger et Gromoll (loc. cit.). Soit $K$ un domaine fondamental pour l'action de $\pi_{1}(M)$ sur $\tilde{M}$. C'est un compact et sa projection $\rho(K)$ sur $V$ est donc un compact dont l'orbite par $\rho\left(\pi_{1}(M)\right)$ est $V$ toute entière. Puisque $V$ est supposée non compacte, il existe en un point $p \in \rho(K)$ un rayon géodésique $\gamma:[0, \infty[\rightarrow V$ (i.e. une demi-droite géodésique) tel que $\gamma(0)=p \in \rho(K)$. Soit $g_{n}$ une suite d'isométries de $\rho\left(\pi_{1}(M)\right)$ telle que $g_{n}(\gamma(n))=$ $p_{n} \in \rho(K)$. La géodésique $\gamma_{n}$ de $V$ définie $\operatorname{par} \gamma_{n}(0)=p_{n}$ et $\gamma_{n}^{\prime}(0)=d g_{n}\left(\gamma^{\prime}(n)\right)$ est donc un rayon géodésique en restriction à $[-n, \infty$ [. Par compacité, on peut extraire une sous-suite $n_{i}$ de sorte que $p_{n_{i}}$ et $\gamma_{n_{i}}^{\prime}(0)$ convergent respectivement vers $p_{0} \in \rho(K)$ et $v_{0} \in T_{p_{0}}^{1}(V)$. Par construction, la géodésique passant en $p_{0}$ à la vitesse $v$ est une droite géodésique.

Lemme 3.4. - L'action de $\pi_{1}(M)$ sur $\mathbb{D}$ est discrète.

Puisque le groupe des isométries de $V$ est fini ([1]), on est ramené à considérer un groupe $H$ dont l'action est libre, discrète, diagonale, cocompacte et isométrique sur le produit $\mathbb{C}^{l} \times \mathbb{D}$. Il reste à voir que cette action reste discrète sur le second facteur, auquel cas chaque feuille de $\mathcal{F}$ sera fermée, situation décrite par l'exemple iii) du théorème 1.2.

Ceci va résulter du fait général suivant :

THÉORÈMe 3.5 (Auslander, [13, p. 149]). - Soient $G$ un groupe de Lie et $R$ un sous-groupe connexe, résoluble et distingué. Soit $\pi: G \rightarrow G / R$ la projection canonique. Soit $H$ un sousgroupe fermé de $G$ dont la composante neutre $H^{0}$ est résoluble. Alors la composante neutre de $\overline{\pi(H)}$ est résoluble.

Preuve du lemme 3.4. - Il suffit manifestement de montrer que $H$, vu comme réseau de $\mathbb{C}^{l} \times U(l) \times S l(2, \mathbb{R})$ se projette sur un réseau de $S l(2, \mathbb{R})$. On note $H_{1}$ l'image de $H$ par cette projection ; remarquons que $H_{1}$ n'est pas conjugué à un sous-groupe triangulaire de $S l(2, \mathbb{R})$ car $\mathbb{C}^{l} \times \mathbb{D} / H$ est de première classe de Chern non nulle. Par conséquent, $H_{1}$ est, soit un réseau, soit dense dans $S l(2, \mathbb{R})$. Supposons par l'absurde que $\overline{H_{1}}=S l(2, \mathbb{R})$. Notons $\pi$ et $\pi_{1}$ les projections canoniques respectives de $\mathbb{C}^{l} \times U(l) \times S l(2, \mathbb{R})$ sur $U(l) \times S l(2, \mathbb{R})$ et de $U(l) \times S l(2, \mathbb{R})$ sur $S l(2, \mathbb{R})$. D'après le théorème d'Auslander, la composante neutre $\Gamma$ de 
$\overline{\pi(H)}$ est résoluble; par suite, $\pi_{1}(\Gamma)$ est un sous-groupe (non nécessairement fermé) résoluble de $S l(2, \mathbb{R})$. Par hypothèse, l'adhérence de $\pi_{1}(\pi(H))$ est précisément $S l(2, \mathbb{R})$; ceci entraîne alors que $\overline{\pi(H)}$ admet une infinité de composantes connexes $\left(\Gamma_{n}\right)_{n \in \mathbb{N}}\left(\Gamma_{0}=\Gamma\right)$ telles que $\bigcup_{n \in \mathbb{N}} \pi_{1}\left(\Gamma_{n}\right)$ soit dense dans $S l(2, \mathbb{R})$. Par compacité de $U(l)$, on a $\pi_{1}(\overline{\pi(H)})=S l(2, \mathbb{R})$, mais ceci est impossible vu que $S l(2, \mathbb{R})$ ne peut s'exprimer comme union dénombrable de translatés d'un sous-groupe résoluble.

Remarque 3.6. - On pourra observer que la proposition 3.1, et donc la conclusion que $\mathcal{F}$ est une fibration, restent valides sans supposer l'existence a priori d'un feuilletage en courbes hyperbolique transverse (ici $\mathcal{C}_{\omega}$ ). En fait, seul importe dans la démonstration de la proposition 3.1 l'existence sur $N_{\mathcal{F}}$ d'une métrique lisse $\mathcal{F}$ invariante par holonomie de $\mathcal{F}$ et à courbure strictement négative.

Cette remarque pourra s'avérer utile dans la section 4 que nous allons maintenant aborder.

\section{Les feuilletages $\mathcal{F}$ et $\mathcal{C}_{\omega}$ sont supposés tangents}

En d'autres termes, les courbes intégrales du feuilletage en courbe $\mathcal{C}_{\omega}$ sont tracées dans les feuilles de $\mathcal{F}$.

\subsection{Pseudo-effectivité du fibré canonique de $M$}

Nous utiliserons largement les travaux de Brunella qui étendent, dans le cadre kählérien, certains des résultats de semi-positivité établis par Miyaoka pour les variétés projectives, et dont une version récente est présentée dans [5].

Lemme 4.1. - Le feuilletage $\mathcal{C}_{\omega}$ n'est pas un feuilletage en courbes rationnelles.

Nous adoptons dans l'énoncé de ce lemme et sa preuve la terminologie utilisée par Brunella $([4,5])$.

Un feuilletage en courbe $\mathcal{C}$ sur une variété $X$ est dit rationnel (ou à courbes rationnelles) si, par tout point de $X$ passe une courbe rationnelle tangente à $\mathcal{C}$.

De plus, lorsque ce feuilletage est une fibration (localement triviale) en restriction à un ouvert de Zariski $X_{0}$ de $X$, on dit que $\mathcal{C}$ est une quasi-fibration rationnelle.

D'un point de vue birationnel, remarquons qu'il n'y a pas lieu de distinguer ces deux cas puisqu'on passe du premier au second par une succession d'éclatements.

Preuve du lemme 4.1. - Supposons par l'absurde que $\mathcal{C}_{\omega}$ soit rationnel. Il s'agit d'exploiter le fait qu'on peut déformer les courbes intégrales de $\mathcal{C}_{\omega}$ tout en restant tangent à $\mathcal{F}$, ce qui devrait entraîner la «négativité » du fibré $K_{\mathcal{F}}$ et contredire ainsi nos hypothèses.

Soit $\pi: X \rightarrow M$ une application obtenue par composition d'éclatements de centres lisses. Notons $\widetilde{\mathcal{C}_{\omega}}$ et $\widetilde{\mathcal{F}}$ les feuilletages pull-back respectifs de $\mathcal{C}_{\omega}$ et $\mathcal{F}$ par $\pi$, cette dernière application étant choisie de telle sorte que $\widetilde{\mathcal{C}_{\omega}}$ soit une fibration quasi-rationnelle.

On désigne par $p: X_{0} \rightarrow B=X_{0} / \widetilde{C_{\omega}}$ la fibration correspondante.

Remarquons que $\widetilde{\mathcal{F}}$ n'est plus nécessairement régulier; en revanche, $\widetilde{\mathcal{C}_{\omega}}$ reste tangent à $\widetilde{\mathcal{F}}$ et ce dernier se redescend donc en un feuilletage $\mathcal{F}_{1}$ de codimension 1 sur la base $B$.

Soient $\mathcal{L}_{1}$ une feuille de $\mathcal{F}_{1}$ et $U_{1}$ un ouvert de $\mathcal{F}_{1}$ biholomorphe à un polydisque.

$4^{\mathrm{e}}$ SÉRIE - TOME $41-2008-\mathrm{N}^{\mathrm{o}} 4$ 
D'un tel choix, il résulte que $p^{-1}\left(U_{1}\right)$ est tangent à $\widetilde{\mathcal{F}}$ et la fibration induite au-dessus de $U_{1}$ est holomorphiquement triviale : $p^{-1}\left(U_{1}\right) \simeq U_{1} \times \mathbb{P}^{1}$. Par suite, sur chaque fibre $F\left(\simeq \mathbb{P}^{1}\right)$ de $p^{-1}\left(U_{1}\right)$, on obtient

$$
T p^{-1}\left(U_{1}\right)_{\mid F}=\bigoplus_{i=1}^{n-2} \mathcal{O}(0) \oplus \mathcal{O}(2) .
$$

Considérons maintenant une section holomorphe non triviale $\xi$ de $\Omega_{M}^{n-1} / \Omega_{\mathcal{F}}^{n-1}(L)$ où $\Omega_{\mathcal{F}}^{n-1}$ désigne le faisceau des germes des $n-1$ formes différentielles holomorphes nulles en restriction aux feuilles de $\mathcal{F}$ et $L=$ Dét $\mathcal{F}$ est par hypothèse plat.

En tirant en arrière par $\pi$, on récupère une section non triviale de $\Omega_{X}^{n-1} / \Omega_{\tilde{\mathcal{F}}}^{n-1}\left(\pi^{*} L\right)$ qui induit par restriction une section de $\wedge^{n-1} T^{*} p^{-1}\left(U_{1}\right)_{\mid F}$. Cette section est non nulle si $F$ est correctement choisie, ce qui donne la contradiction recherchée, vu que, d'après (4.1), on a $\wedge^{n-1} T^{*} p^{-1}\left(U_{1}\right)_{\mid F}=\mathcal{O}(-2)$.

Proposition 4.2. - Le fibré canonique $K_{M}$ de la variété $M$ est pseudo-effectif.

Rappelons qu'un fibré en droite $L$ sur $X$ kählérienne compacte est dit pseudo-effectif si $c_{1}(L)$ peut être représentée par un courant positif fermé de bidegré $(1,1)$, on dit alors que $c_{1}(L)$ est une classe pseudo-effective.

Démonstration. - On a montré (lemme 4.1) que $\mathcal{C}_{\omega}$ n'est pas un feuilletage en courbes rationnelles. Dans ces conditions, Brunella [5] a établi que le fibré cotangent $T_{\mathcal{C}_{\omega}}^{*}$ de $\mathcal{C}_{\omega}$ est pseudo-effectif. Par ailleurs, la $n-1$ forme $\omega$ est holomorphe à valeurs dans un fibré plat; par adjonction, on obtient alors que $c_{1}\left(K_{M}\right)=c_{1}(D)+c_{1}\left(T_{\mathcal{C}_{\omega}}^{*}\right)$ où $D$ désigne le diviseur effectif des zéros de $\omega$. On conclut en remarquant qu'une somme de classes pseudo-effectives reste pseudo-effective.

Rappelons qu'en combinant la formule d'adjonction (1.1) et le fait que $K_{\mathcal{F}}$ est supposé numériquement trivial, on obtient que

$$
c_{1}\left(N_{\mathcal{F}^{*}}^{*}\right)=c_{1}\left(K_{M}\right)=-c_{1}(M) .
$$

En particulier la proposition 4.2 implique la pseudo-effectivité du fibré conormal $N_{\mathcal{F}}{ }^{*}$.

On est alors dans les conditions d'application du lemme qui suit, démontré par Brunella [3] dans le cadre des feuilletages sur les surfaces complexes compactes, mais qui s'adapte sans difficultés lorsque la variété ambiante est kählérienne. Nous en donnerons la démonstration par commodité pour le lecteur (voir aussi [6]).

Lemme 4.3. - Soit $\mathcal{F}$ un feuilletage holomorphe de codimension 1 sur $M$ kählérienne compacte. Supposons que $N_{\mathcal{F}}{ }^{*}$ soit pseudo-effectif; alors il existe un courant positif fermé de bidegré $(1,1)$ invariant par holonomie de $\mathcal{F}$.

Démonstration. - Par hypothèse, le fibré $N_{\mathcal{F}}{ }^{*}$ admet une métrique singulière dont les poids locaux sont plurisousharmoniques. La forme de courbure de cette métrique est donc donnée par un courant positif $T$ localement défini par $T=\frac{\sqrt{-1}}{2 \pi} \partial \bar{\partial} F$, où $F$ est un poids local de la métrique. On peut choisir ces poids locaux de façon à obtenir sur $M$ une forme $\eta$ (a priori non lisse) telle que, localement :

$$
\eta=\sqrt{-1} e^{2 F} d f \wedge d \bar{f}
$$


où $\{d f=0\}$ est une équation locale de $\mathcal{F}$. Soit $\theta$ une forme de Kähler; on déduit du théorème de Stokes (pour les courants) que

$$
\int_{M} \partial \bar{\partial} \eta \wedge \theta^{n-2}=0
$$

D'autre part, le courant $\partial \bar{\partial} \eta$ est positif (l'exponentielle d'une fonction $p s h$ est $p s h$ ). La nullité de l'intégrale précédente implique alors que $e^{F}$ est pluriharmonique et donc constante dans les feuilles.

L'existence d'un tel courant invariant par holonomie confère au feuilletage $\mathcal{F}$ des propriétés qualitativement semblables à celles des feuilletages riemanniens. Plus précisément, il est montré dans [3, p. 590-592] que $\mathcal{F}$ entre dans la catégorie des feuilletages quasiuniformément isométriques étudiés par Kellum ([K]). Suivant Brunella (loc.cit.), on peut alors affirmer que $M$ est union disjointe de minimaux de $\mathcal{F}$, chaque minimal étant soit une feuille compacte, soit une sous-variété topologique de codimension 1 réelle, soit $M$.

Nous allons maintenant décortiquer toutes les configurations envisageables dans les sections à venir.

\subsection{Le feuilletage $\mathcal{F}$ est minimal}

En d'autres termes, chaque feuille est dense dans $M$.

Proposition 4.4. - Sous les hypothèses de 4.2, $\mathcal{F}$ est donnée par l'exemple i) du théorème 1.2 ( «feuilletage linéaire sur le tore»).

Démonstration. - Rappelons (preuve du lemme 4.3) qu'il existe une métrique transverse au feuilletage $\mathcal{F}$, invariante par holonomie et s'écrivant localement sous la forme

$$
\eta=\sqrt{-1} e^{2 F} d f \wedge d \bar{f}
$$

et dont la forme de courbure $-T\left(T=\frac{\sqrt{-1}}{2 \pi} \partial \bar{\partial} F\right)$ est un courant représentant

$$
-c_{1}\left(N^{*} \mathcal{F}\right)=c_{1}(M) \text {. }
$$

Si le feuilletage est minimal, on obtient par transitivité (voir [3] pour les détails) que $N_{\mathcal{F}}$ admet une métrique lisse transverse invariante par holonomie et de courbure de Gauss $\kappa$ constante. Par pseudo-effectivité de $N_{\mathcal{F}}{ }^{*}$, on a nécessairement $\kappa \leq 0$. Le cas $\kappa<0$ est en fait exclu (remarque 3.6). Par suite, cette métrique est plate, ce qui, compte tenu de nos hypothèses sur $K_{\mathcal{F}}$, signifie que $c_{1}(M)=0$. On conclut alors par la remarque 2.1.

\subsection{Chaque feuille de $\mathcal{F}$ est compacte}

Il n'y a rien à démontrer : $\mathcal{F}$ est alors une fibration et c'est l'exemple iii) du théorème 1.2.

Il reste à étudier les feuilletages comportant un minimal de codimension 1 réelle. Ceux-ci sont décrits comme suit [3] :

a) l'adhérence de chaque feuille est de codimension 1 réelle;

b) il y a exactement deux feuilles compactes et les autres feuilles ont des adhérences de codimension 1 réelle.

Avant d'étudier plus en détail ces deux cas, nous allons formuler de manière plus précise les résultats obtenus par Kellum [8]. 


\subsection{Structure du pseudo-groupe d'holonomie}

Rappelons un des principaux résultats de Kellum : dans le cadre quasi-isométrique, l'adhérence $\bar{H}$ du pseudo-groupe d'holonomie $H$ est un pseudo-groupe de Lie. En particulier, on hérite d'un faisceau localement constant $\mathcal{A}$ de germes de champs de vecteurs basiques qui est précisément, en chaque point de $M$, formé de l'algèbre de Lie réelle des générateurs infinitésimaux de $\bar{H}$.

D'après [11, prop. 4.8, p. 171], cette algèbre de Lie est en fait engendrée (sur $\mathbb{R}$ ) par des champs de vecteurs holomorphes si le feuilletage est lui-même holomorphe.

Le lemme qui suit détaille la structure de $\mathcal{A}_{x}$ en un point $x$ de $M$.

Lemme 4.5. - Sous les hypothèses a) ou b) précédentes, l'algèbre de Lie $\mathcal{A}_{x}$ est de dimension réelle 1 en chaque point $x \in M$.

De plus, dans une coordonnée holomorphe $z$ transverse au feuilletage $\mathcal{F}$ correctement choisie et telle que $z(x)=0$, un générateur de $\mathcal{A}_{x}$ s'écrit sous la forme

ou

$$
X(z)=\frac{\partial}{\partial z}
$$

$$
X(z)=2 i \pi \alpha z \frac{\partial}{\partial z}, \alpha \in \mathbb{R} .
$$

Démonstration. - Fixons $x \in M$ et notons $\overline{\mathcal{F}_{x}}$ l'adhérence de la feuille passant par $x$. Soit $\{d z=0\}$ une équation locale de $\mathcal{F}$ avec $z(x)=0$. Les éléments de $\mathcal{A}_{x}$ peuvent se voir comme des champs de vecteurs définis sur une petite transversale $T$ à $\mathcal{F}$ paramétrée par $z$. Considérons maintenant la sous-algèbre de $\mathcal{A}_{x}$ définie par

$$
\mathcal{A}_{x}^{0}=\left\{\mathcal{V} \in \mathcal{A}_{x}, \mathcal{V}(x)=0\right\}
$$

Examinons d'abord le cas où $\mathcal{A}_{x}^{0}$ est réduit à $\{0\}$. Soit $X \in \mathcal{A}_{x} \backslash\{0\}$. Pour un choix adéquat de $z$, on peut mettre ce champ de vecteurs sous la forme

$$
X(z)=\frac{\partial}{\partial z}
$$

dont les courbes intégrales (pour le flot réel) sont alors les droites horizontales de T. Par conséquent, $\overline{\mathcal{F}_{x}}$ est de codimension 1 réelle et tout autre élément de $\mathcal{A}_{x} \backslash\{0\}$ a les mêmes courbes intégrales que $X$.

On en déduit que

$$
\mathcal{A}_{x}=\mathbb{R} \frac{\partial}{\partial z} .
$$

Supposons maintenant que $\mathcal{A}_{x}^{0} \neq\{0\}$. On peut en extraire un champ de vecteurs non trivial qui s'écrit, pour un choix convenable de $z$, sous forme normale : $X_{\alpha}(z)=2 i \pi \alpha z \frac{\partial}{\partial z}$ ou $X_{\nu, \lambda}(z)=\frac{z^{\nu}}{1+\lambda z^{\nu}} \frac{\partial}{\partial z}$ avec $\alpha, \lambda \in \mathbb{C}$ et $\nu \in \mathbb{N} \backslash\{0\}$ (voir par exemple [12]). Supposons d'abord que $X=X_{\alpha}$ avec $\alpha \notin \mathbb{R}$. Dans ce cas (quitte à prendre $-X$ ), l'origine est un foyer stable (dynamique spiralante) et il existe un compact $K \in T$ d'intérieur non vide tel que pour tout $t \in\left[0,+\infty\left[, \exp t X(K) \in T\right.\right.$ et $\lim _{t \rightarrow+\infty} \operatorname{Vol}(\exp t X(K))=0$ où Vol désigne le volume euclidien. Cette propriété est visiblement incompatible avec le fait que $\mathcal{F}$ est quasiuniformément isométrique.

L'existence d'un tel compact $K$ est aussi garantie dans le cas où $X=X_{\nu, \lambda}$ (prendre un domaine de type «sectoriel» où la dynamique de $X$ est attractive). 
Par suite, on a nécessairement $X=X_{\alpha}$ avec $\alpha \in \mathbb{R}$. Le flot réel est alors un groupe à un paramètre de rotations; ainsi, pour tout point $y \in M$ assez proche de $x(y \neq x), \overline{\mathcal{F}_{y}}$ est de codimension 1 réelle. De même que précédemment, on conclut alors que

$$
\mathcal{A}_{x}=\mathbb{R} 2 i \pi \alpha z \frac{\partial}{\partial z}, \alpha \in \mathbb{R} .
$$

Définition 4.6. - Soit $x \in M$. On dira que $\mathcal{A}_{x}$ est :

- de type (1) si $\mathcal{A}_{x}=\mathbb{R} \frac{\partial}{\partial z}$;

- de type (2) si $\mathcal{A}_{x}=\mathbb{R} 2 i \pi \alpha z \frac{\partial}{\partial z}, \alpha \in \mathbb{R}$.

Compte tenu de la description de $\mathcal{A}$, on peut construire une 1 -forme $\eta$ définissant le feuilletage $\mathcal{F}$ de la façon suivante.

Pour un recouvrement suffisamment fin $M=\bigcup_{i \in I} U_{i}$ par des ouverts $U_{i}$ connexes, on peut choisir, pour tout indice $i$ un champ $X_{i} \in \mathcal{A}\left(U_{i}\right)$ non trivial tel que $\mathcal{A}\left(U_{i}\right)=\mathbb{R} X_{i}$. Sur chaque $U_{i}$, il existe une unique forme méromorphe $\eta_{i}$ tangente à $\mathcal{F}$ et telle que $\eta_{i}\left(X_{i}\right)=1$. En outre, sur chaque intersection $U_{i} \cap U_{j}$, on a $\eta_{i}=h_{i j} \eta_{j}, h_{i j} \in \mathcal{O}^{*}\left(U_{i} \cap U_{j}\right)$ localement constants.

La collection des $\eta_{i}$ définit donc une section $\eta$ de $\Lambda^{1}(L)$ où $\Lambda^{1}$ désigne le faisceau des germes de 1-formes méromorphes sur $M$ et $L$ est un fibré en droite plat associé au cocycle multiplicatif $\left(h_{i j}\right)$.

\subsection{L'adhérence de chaque feuille de $\mathcal{F}$ est de codimension 1 réelle}

Il est facile de réaliser ce type de configuration par un feuilletage linéaire sur le tore. La proposition qui suit établit la réciproque.

Proposition 4.7. - Sous les hypothèses de 4.5, $\mathcal{F}$ est donnée par l'exemple i) du théorème 1.2 ( «feuilletage linéaire sur le tore »).

Démonstration. - On reprend les notations de la section 4.4. Sous nos hypothèses, en chaque point $x$ de $M$, l'algèbre de Lie $\mathcal{A}_{x}$ est clairement du type (1). En particulier, la forme $\eta$ ne comporte pas de diviseurs de pôles ou de zéros. Par suite $N_{\mathcal{F}}{ }^{*}=L^{*}$ et $c_{1}\left(N_{\mathcal{F}}^{*}\right)=-c_{1}(M)=0$. On conclut alors par la remarque 2.1.

\subsection{Le feuilletage $\mathcal{F}$ admet deux feuilles compactes et les autres feuilles ont des adhérences de codimension 1 réelle}

Démonstration. - Il s'agit de voir que ce cas ne se produit pas.

Supposons par l'absurde qu'il existe un tel feuilletage. Le long de chaque feuille compacte $H_{i}(i=1,2), \mathcal{A}_{x}$ est manifestement du type (2) et du type (1) ailleurs. Par suite, $\eta$ est à pôles simples le long des feuilles compactes et sans diviseurs de zéros. Il en résulte que $c_{1}\left(N_{\mathcal{F}}{ }^{*}\right)$ est représentée par le courant

$$
T=-\delta_{H_{1}}-\delta_{H_{2}} .
$$

Donc, si $\theta$ désigne une forme de Kähler, on doit avoir $c_{1}\left(N_{\mathcal{F}^{*}}^{*}\right) \cdot\left[\theta^{n-1}\right]=-\sum_{i=1}^{2} \int_{H_{i}} \theta^{n-1}<0$, ce qui contredit la pseudo-effectivité de $N_{\mathcal{F}}{ }^{*}$ (établie en 4.1). 


\section{Remerciements}

L'auteur exprime sa gratitude à Frank Loray et Yves Guivarc'h pour lui avoir communiqué un certain nombre de références et remercie vivement le rapporteur pour ses précieux commentaires et suggestions.

\section{RÉFÉRENCES}

[1] A. Beauville, Variétés kähleriennes dont la première classe de Chern est nulle, J. Diff. Geom. 18 (1983), 755-782.

[2] F. A. Bogomolov, Kähler manifolds with trivial canonical class, Izv. Akad. Nauk SSSR Ser. Mat. 38 (1974), 11-21.

[3] M. Brunella, Feuilletages holomorphes sur les surfaces complexes compactes, Ann. Sci. École Norm. Sup. 30 (1997), 569-594.

[4] M. Brunella, Plurisubharmonic variation of the leafwise Poincaré metric, Internat. J. Math. 14 (2003), 139-151.

[5] M. Brunella, A positivity property for foliations on compact Kähler manifolds, Internat. J. Math. 17 (2006), 35-43.

[6] M. Brunella, J. V. Pereira, F. Touzet, Kähler manifolds with split tangent bundle, Bull. Soc. Math. France 134 (2006), 241-252.

[7] J. Cheeger, D. Gromoll, The splitting theorem for manifolds of nonnegative Ricci curvature, J. Diff. Geom. 6 (1971/72), 119-128.

[8] M. Kellum, Uniformly quasi-isometric foliations, Ergodic Theory Dynam. Systems 13 (1993), 101-122.

[9] S. Kobayashi, K. Nomizu, Foundations of differential geometry. Vol. II, John Wiley \& Sons Inc., 1996.

[10] S. Kobayashi, H. Wu, Complex differential geometry, DMV Seminar 3, Birkhäuser, 1987.

[11] F. Loray, J. C. Rebelo, Minimal, rigid foliations by curves on $\mathbb{C P}^{n}$, J. Eur. Math. Soc. 5 (2003), 147-201.

[12] J. Martinet, J.-P. Ramis, Classification analytique des équations différentielles non linéaires résonnantes du premier ordre, Ann. Sci. École Norm. Sup. 16 (1983), 571621.

[13] M. S. Raghunathan, Discrete subgroups of Lie groups, Ergebnisse der Mathematik und ihrer Grenzgebiete, Band 68, Springer, 1972. 
[14] S. T. YAU, On the Ricci curvature of a compact Kähler manifold and the complex Monge-Ampère equation. I, Comm. Pure Appl. Math. 31 (1978), 339-411.

(Manuscrit reçu le 6 novembre 2007 ; accepté, après révision, le 21 février 2008.)

Frédéric TouzeT

Université de Rennes 1

Institut de Recherche Mathématique de Rennes

Campus de Beaulieu

35042 Rennes Cedex, France

E-mail: frédéric.touzet@univ-rennes1.fr 